\title{
DEFINITIONS OF THE TIDAL INTERACTION AND MERGER PHENOMENA
}

A panel on these issues was held with G. Burbidge as moderator and contributions from T. Chatterjee, T. Heckman, J. Hutchings, M. Noguchi, S. Simkin, and W. Keel (who prepared this summary).

\section{Tidal interactions:}

Several panelists stressed the need for care in defining observational criteria for considering a system interacting, beyond some kind of morphological peculiarity. Inevitably, some theoretical guidance is needed in this kind of interpretation. For example, narrow bridges and tails are well understood as results of certain kinds of interactions involving disk systems. Heckman stressed that such features should be seen in the old stellar population (the "Crimson Tide" approach) to avoid confusion with line emission or modest amounts of recent star formation that might have been influenced by hydrodynamical processes. Systems of these kinds will generally have an identifiable companion, perhaps tidally stripped or with its own tidal distortions, allowing further consistency checks on an interaction.

For ellipticals, there has been considerable success in modelling the broad tidal distortions characteristic of the high stellar velocity dispersions found in these galaxies. Further evidence of interaction with disk systems, or gas-rich companions, is made possible by this large velocity dispersion and the almost total lack of cool interstellar matter in ellipticals - stars from a cannibalized disk will form shells or rings, and substantial amounts of H I or dust are often associated with other indicators of interaction, such as environment or morphology. Note that these indicators require the (rare?) occurrence of significant mass transfer between two interacting systems.

The situation for AGNs and QSOs is less clear. As noted in Heckman's review, it is not clear what population we should compare QSO host galaxies with. Simkin proposed that in some kinds of apparent double systems, the mere fact of the presence of an active nucleus raises the probability that we are observing a physically interacting double.

\section{Mergers:}

The degree of certainty about defining a galaxy merger depends strongly on when we observe it. Early on, two nuclei may still be distinct; note that some care may be needed to avoid confusion of a region of rapid star formation, but dynamically insignificant mass, with a genuine galactic nucleus. When the nuclei can no longer be distinguished, more circumstantial tracers such as shells or H I in ellipticals must be used. The discovery of counter-rotating systems of stars or gas in several E and S0 systems in recent years is very interesting in this connection. In disk systems, early-type spirals sometimes seem capable of exhibiting shells after accretion of a dynamically cold companion, and disturbance of the disk velocity field (or global H I profile) may be observable when there is little morphological trace of the merger. When AGNs are involved, one can seek to measure an externally imposed tidal torque via precession of radio jets.

The frequent associations of starbursts and mergers of various kinds leads to the possibility of associating disturbed galaxies with strong bursts uniquely with mergers, 
though there is a serious risk of presupposing the effect to be measured. A potentially promising technique for tracing the fraction of $\mathrm{E} / \mathrm{S} 0$ systems that are products of disk mergers is through globular-cluster counts, since the specific frequency of globulars depends on morphological type of the original host system.

\section{Theoretical Issues:}

Noguchi discussed the possibility that star formation may occur in different regimes in interacting and merging galaxies, driven by changes in the dominant kinds of cloud collisions. Collision velocities in a tidally perturbed disk will be relatively modest (of order $10 \mathrm{~km} \mathrm{~s}^{-1}$, brought on by crossing of nearby orbits ("intra-galactic collisions"). In mergers, some "inter-galactic" collisions should have much higher velocities, as material originally part of different galaxies comes into contact as fast as several hundred $\mathrm{km} \mathrm{s}^{-1}$. Since cloud disruption is likely for most such fast collisions, differences in the resulting star formation might be detectable.

Chatterjee addressed the expected relative frequencies of tidal encounters and mergers, pointing out that most mergers must take place within 2-3 times the orbital period shortly before mergers. If mergers take much longer, the statistics of pairs and apparent merger remnants would be violated. This may require the common presence of massive halos in systems merging at the present epoch.

\section{Unresolved Questions:}

How large a tidal event should we regard as "significant"?

How many early-type galaxies are merger products?

What is the evolution of interaction and merger rates with cosmic time? 